\title{
Statefinders, higher-order energy conditions, and sudden future singularities.
}

\author{
Mariusz P. Dąbrowsk* \\ Institute of Physics, University of Szczecin, \\ Wielkopolska 15, 70-451 Szczecin, Poland.
}

(Dated: November 9, 2018)

\begin{abstract}
We link observational parameters such as the deceleration parameter, the jerk, the kerk (snap) and higher-order derivatives of the scale factor, called statefinders, to the conditions which allow to develop sudden future singularities of pressure with finite energy density. In this context, and within the framework of Friedmann cosmology, we also propose higher-order energy conditions which relate time derivatives of the energy density and pressure which may be useful in general relativity.
\end{abstract}

PACS numbers: 98.80.Jk,98.80.Es,04.20.Ha

*Electronic address: mpdabfz@sus.univ.szczecin.pl 
The current observational data from type Ia supernovae 1] favours the cosmological models of dark energy of the phantom type [2]. Phantom is the matter which has a very strong negative pressure which violates the null energy condition $\varrho+p>0(p$ - the pressure, $\varrho$ - the energy density) and, consequently, all the remaining energy conditions [3]. It has very severe physical consequences since it leads to classical and quantum instabilities [4]. It is even argued that it should not be accepted and that there should be some other explanation of the phenomenon of dimming of supernovae [5]. On the other hand, the observational data is interpreted only in terms of the simple framework of Friedmann cosmology with an additional restriction of the barotropic form of the equation of state $p=w \varrho(w=$ const.) which links the pressure and the energy density of matter. However, in general, the equation of state does not necessarily have to be of barotropic type. The most interesting possibility is that it changes with time during the evolution of the universe which makes the barotropic index $w=w(t)$ to be the function of time [6, 7].

From the observational point of view one is able to expand the equation of state in series around the present moment of the evolution by taking appropriate derivatives of the pressure with respect to the energy density. This procedure, however, requires the usage of the time derivatives of the scale factor up to suitable orders which would show the "tendency" of the universe to change its equation of state in future or study its evolution in the past. Due to this, the observational parameters made of the time derivatives of the scale factor were named statefinders [8]. Of course, in a general case, the spatial derivatives of all the kinematic quantities in non-isotropic models may also be involved, but in this paper we restrict ourselves to isotropic models only. In fact, statefinders generalize such wellknown observational characteristics of the expansion as the Hubble (first-order) and the deceleration (second-order) parameters. The third-order characteristics was called jerk [8] while the fourth-order characteristic was called either snap [9] or kerk [10]. In this paper we discuss even higher-order characteristics with the suggested names "lerk", "merk" etc. with the logical numbering them alphabetically with an addition of the term "erk" [10].

Phantom models of the universe $(w<-1)$ admit a new type of singularity which is called Big-Rip (BR) 2]. At Big-Rip the energy density and pressure diverge as a result of having the infinite value of the scale factor $a(t)$ at finite time. This is different from the ordinary Big-Crunch (BC) singularity which leads to a blow-up of the energy density and pressure for the scale factor approaching zero value at finite time. Due to time symmetry 
the Big-Rip time singularity could have also appeared in the past but it is of course different from ordinary Big-Bang (BB) singularity.

However, if one drops the assumption of an exact equation of state in the field equations one is able to face another type of singularity in the universe, which is called a sudden future (SF) singularity [11, 12, 13, 14]. This is a singularity of pressure only, with finite energy density of matter. Unlike phantom, it appears for the matter fulfilling the strong energy condition $\varrho>0, \varrho+3 p>0$, the weak energy condition $\varrho>0, \varrho+p>0$, though violating the dominant energy condition $\varrho>0,-\varrho<p<\varrho\lfloor 15,16,17]$. The nature of SF singularities in terms of geodesic completeness has also been discussed [18].

In this paper we discuss SF singularities [11, 12] and generalized SF singularities [16] and relate their emergence to observational parameters composed of higher-order derivatives of the scale factor. These, on the other hand, may give some insight into the problem of the (changing in time) equation of state of the matter in the universe, since they are directly related to higher-order time derivatives of the energy density and pressure according to the expansion

$$
\begin{aligned}
p & =p_{0}+\left.\frac{d p}{d \varrho}\right|_{0}\left(\varrho-\varrho_{0}\right)+\left.\frac{1}{2 !} \frac{d^{2} p}{d \varrho^{2}}\right|_{0}\left(\varrho-\varrho_{0}\right)^{2}+O\left[\left(\varrho-\varrho_{0}\right)^{3}\right] \\
& =p_{0}+\frac{\dot{p}_{0}}{\dot{\varrho}_{0}}\left(\varrho-\varrho_{0}\right)+\frac{1}{2 !} \frac{\ddot{p}_{0} \dot{\varrho}_{0}-\dot{p}_{0} \ddot{\varrho}_{0}}{\dot{\varrho}^{3}}\left(\varrho-\varrho_{0}\right)^{2}+O\left[\left(\varrho-\varrho_{0}\right)^{3}\right],
\end{aligned}
$$

where index " 0 " refers to a quantity taken at the current moment of the evolution $t=t_{0}$.

The SF singularities appear in the simple framework of Einstein-Friedmann cosmology governed by the standard field equations (we have assumed that $8 \pi G=c=1$ )

$$
\begin{aligned}
& \varrho=3\left(\frac{\dot{a}^{2}}{a^{2}}+\frac{K}{a^{2}}\right), \\
& p=-\left(2 \frac{\ddot{a}}{a}+\frac{\dot{a}^{2}}{a^{2}}+\frac{K}{a^{2}}\right) .
\end{aligned}
$$

together with the energy-momentum conservation law

$$
\dot{\varrho}=-3 \frac{\dot{a}}{a}(\varrho+p),
$$

$a(t)$ is the scale factor, $K=0, \pm 1$ is the curvature index, $\varrho$ is the energy density, and $p$ is the pressure.

From (3) one can see [11] that the singularity of pressure $p \rightarrow \infty$ occurs when acceleration $\ddot{a} \rightarrow-\infty$, while $a$ and $\dot{a}$ are regular. This can be achieved for the scale factor of the form

$$
a(t)=A+\left(a_{s}-A\right)\left(\frac{t}{t_{s}}\right)^{m}-A\left(1-\frac{t}{t_{s}}\right)^{n},
$$


where $a_{s} \equiv a\left(t_{s}\right)$ with $t_{s}$ being the SF singularity time and $A, m, n=$ const. It is obvious from (5) that $a(0)=0$ and so at zero of time a BB singularity develops. For the sake of further considerations it is useful to write down the higher-order derivatives of the scale factor (5), i.e.,

$$
\begin{aligned}
\dot{a} & =\frac{m}{t_{s}}\left(a_{s}-A\right)\left(\frac{t}{t_{s}}\right)^{m-1}+A \frac{n}{t_{s}}\left(1-\frac{t}{t_{s}}\right)^{n-1}, \\
\ddot{a} & =\frac{m(m-1)}{t_{s}^{2}}\left(a_{s}-A\right)\left(\frac{t}{t_{s}}\right)^{m-2} \\
& -A \frac{n(n-1)}{t_{s}^{2}}\left(1-\frac{t}{t_{s}}\right)^{n-2}, \\
\dddot{a} & =\frac{m(m-1)(m-2)}{t_{s}^{3}}\left(a_{s}-A\right)\left(\frac{t}{t_{s}}\right)^{m-3} \\
& +A \frac{n(n-1)(n-2)}{t_{s}^{3}}\left(1-\frac{t}{t_{s}}\right)^{n-3},
\end{aligned}
$$

or, for a general time derivative of an order $r$ :

$$
\begin{aligned}
a^{(r)} & =\frac{m(m-1)(m-2) \ldots(m-r+1)}{t_{s}^{r}}\left(a_{s}-A\right)\left(\frac{t}{t_{s}}\right)^{m-r} \\
& +(-1)^{r-1} A \frac{n(n-1)(n-2) \ldots(n-r+1)}{t_{s}^{r}}\left(1-\frac{t}{t_{s}}\right)^{n-r}
\end{aligned}
$$

The main point is that the evolution of the Universe, as described by the scale factor (5), begins with the standard $\mathrm{BB}$ singularity at $t=0$ and terminates at SF singularity for $t=t_{s}$ provided we choose

$$
1<n<2, \quad 0<m \leq 1
$$

For these values of $n$ and $m$ the scale factor (5) vanishes and its derivatives (66)-(17) diverge at $t=0$ leading to the divergence of $\varrho$ and $p$ in (22)-(3) (BB singularity). On the other hand, the scale factor (5) and its first derivative (6) remain constant while its second derivative (7) diverge leading to a divergence of pressure in (3) only with finite energy density (2). It has been shown [16] that more general sudden future singularities appear, provided we choose

$$
N<n<N+1, \quad 0<m \leq 1
$$

instead of (10). It means that for any integer $N+1=r$ we have a singularity in the scale factor derivative $a^{(r)}$ (cf. (9) ) , and consequently in the appropriate pressure derivative $p^{(r-2)}$. 
This, for any $r \geq 3$, gives a sudden future singularity which fulfills all the energy conditions including the dominant one [15].

Now, we introduce the higher-order characteristics or statefinders [8, 9, 10] and relate them to the emergence of sudden future singularities. We also formulate the higher-order energy conditions which may be useful in general relativity.

The well-known characteristics of the universe expansion are: the Hubble parameter

$$
H=\frac{\dot{a}}{a}
$$

and the deceleration parameter

$$
q=-\frac{1}{H^{2}} \frac{\ddot{a}}{a}=-\frac{\ddot{a} a}{\dot{a}^{2}}
$$

while the new characteristics are: the jerk parameter [8]

$$
j=\frac{1}{H^{3}} \frac{\dddot{a}}{a}=\frac{\dddot{a} a^{2}}{\dot{a}^{3}},
$$

and the "kerk" (snap) parameter [9, 10]

$$
k=-\frac{1}{H^{4}} \frac{\dddot{a}}{a}=-\frac{\dddot{a} a^{3}}{\dot{a}^{4}},
$$

the "lerk" parameter

$$
l=\frac{1}{H^{5}} \frac{a^{(5)}}{a}=\frac{a^{(5)} a^{4}}{\dot{a}^{5}},
$$

and "merk", "nerk", "oerk", "perk" etc. parameters, of which a general term may be expressed as

$$
x^{(i)}=(-1)^{i+1} \frac{1}{H^{i}} \frac{a^{(i)}}{a}=(-1)^{i+1} \frac{a^{(i)} a^{i-1}}{\dot{a}^{i+1}},
$$

and its time derivative reads as

$$
\left(x^{(i)}\right)^{\cdot}=H\left[i(q+1) x^{(i)}-\left(x^{(i+1)}+x^{(i)}\right)\right] .
$$

A possible blow-up of statefinders may easily be linked to an emergence of singularities. In particular, this may be the signals for SF singularities. In fact, it is possible to formulate higher-order energy conditions which may be related to statefinders. We will study this in what follows. 
The application of the definitions of the parameters (12), (13), 14), (15), and (16) gives the following equalities for the time derivatives of the Hubble parameter 10]:

$$
\begin{aligned}
\dot{H} & =-H^{2}(q+1) \\
\ddot{H} & =H^{3}(j+3 q+2), \\
\dddot{H} & =-H^{4}[k+4 j+3 q(q+4)+6], \\
\dddot{H} & =H^{5}[l+5 k+10 j(q+2)+30 q(q+2)+24] .
\end{aligned}
$$

Using the equations (2), (3), (41), (12) and (13) the three energy conditions (weak, strong, and dominant) are equivalent to

$$
\begin{aligned}
\varrho+p & =2 H^{2}\left(q+1+\frac{K}{a^{2} H^{2}}\right)>0, \quad \varrho>0 \\
\varrho-p & =2 H^{2}\left(-q+2+2 \frac{K}{a^{2} H^{2}}\right)>0, \quad \varrho>0 \\
\varrho+3 p & =6 q H^{2}>0, \quad \varrho>0 .
\end{aligned}
$$

In fact, the weak energy condition requires both (23) and (24) to hold. Notice that the application of (2), (3) and (41) gives

$$
\dot{H}=\frac{K}{a^{2}}-\frac{1}{2}(\varrho+p)
$$

which, after further differentiation with respect to time, and the usage of the formulas (19)(22), may give the higher-order weak energy conditions which relate time derivatives of the energy density $\varrho$ and the pressure $p$. These are

$$
\begin{aligned}
\dot{\varrho}+\dot{p}= & -2 H^{3}\left(j+3 q+2+2 \frac{K}{a^{2} H^{2}}\right)>0, \quad \dot{\varrho}>0 \\
\ddot{\varrho}+\ddot{p}= & 2 H^{4}[k+4 j+3 q(q+4)+6 \\
& \left.+2 \frac{K}{a^{2} H^{2}}(q+3)\right]>0, \quad \ddot{\varrho}>0 \\
\dddot{\varrho}+\dddot{p}= & -2 H^{5}[l+5 k+10 j(q+2)+30 q(q+2)+24 \\
& \left.+2 \frac{K}{a^{2} H^{2}}(j+9 q+12)\right]>0, \quad \dddot{\varrho}>0 .
\end{aligned}
$$


The application of (23) and (27) allows to write the time derivatives of pressure

$$
\begin{aligned}
\dot{p}= & 2 H^{3}\left(-j+1+\frac{K}{a^{2} H^{2}}\right) \\
\ddot{p}= & 2 H^{4}\left[k+j-3(q+1)-\frac{K}{a^{2} H^{2}}(q+3)\right], \\
\dddot{p}= & -2 H^{5}\left[l+2 k+j(q-1)-6 q^{2}-21 q-12\right. \\
& \left.-(j+9 q+12) \frac{K}{a^{2} H^{2}}\right] .
\end{aligned}
$$

From (30), (31) and (32) one can see that the consecutive SF singularities as given by the condition (11) are strictly related to a blow-up of the statefinders. In fact, for $N=1$ there is blow-up of the deceleration parameter $q$ ( $p$ diverges), for $N=2$ there is a blow-up of the jerk $j$ ( $\dot{p}$ diverges) etc.

Now we can write down the higher-order dominant and strong energy conditions, i.e,

$$
\begin{aligned}
\dot{\varrho}-\dot{p}= & -2 H^{3}\left(-j+3 q+4+4 \frac{K}{a^{2} H^{2}}\right)>0, \quad \dot{\varrho}>0 \\
\dot{\varrho}+3 \dot{p}= & -6 H^{3}(q+j)>0, \quad \dot{\varrho}>0 \\
\ddot{\varrho}-\ddot{p}= & 2 H^{4}[-k+2 j+3 q(q+6)+12 \\
& \left.+4 \frac{K}{a^{2} H^{2}}(q+3)\right]>0, \quad \ddot{\varrho}>0 \\
\dddot{\varrho}+3 \ddot{p}= & 6 H^{4}[k+2 j+q(q+2)]>0, \quad \ddot{\varrho}>0 \\
\dddot{\varrho}-\dddot{p}= & -2 H^{5}\left[-l+k+2 j(4 q+11)+6\left(7 q^{2}+17 q+8\right)\right. \\
& \left.+4(j+9 q+12) \frac{K}{a^{2} H^{2}}\right]>0, \quad \dddot{\varrho}>0 \\
\dddot{\varrho}+3 \dddot{p}= & -6 H^{5}[l+3 k+2 j(5 q+3)+6 q(q+1)]>0, \quad \dddot{\varrho}>0 .
\end{aligned}
$$

Referring to SF singularities, one can see that it is not possible to fulfil any generalized dominant energy condition if any of statefinders $q, j, k, l$ etc. is singular. This is because in the appropriate expressions (23) and (24), (27) and (33) etc. the signs in front of corresponding statefinders are the opposite.

Having given the higher-order time derivatives of the energy density and pressure related to statefinders, one is able to invent some more sophisticated energy conditions which may be obeyed by presumably singular cosmological models [15, 16]. As an example consider an energy condition

$$
\alpha \varrho>\dot{p},
$$


with $\alpha=$ const., which, after the application of (2) and (30) gives

$$
j>\left(1-\frac{3 \alpha}{2 H}\right)\left(1+\frac{K}{a^{2} H^{2}}\right),
$$

and this may prevent the emergence of SF singularity for $N=2$ in (11).

Finally, the observational determination of the value of jerk by using type Ia supernovae sample has been performed [19] and it claims that $j_{0}>0$. Similar investigations may perhaps also be possible for higher-order characteristics such as kerk/snap, lerk etc. Some hints about that are given in [9].

\section{Acknowledgments.}

I appreciate comments from T. Chiba, S. Nojiri, V.K. Onemli, V. Sahni and H. Štefančić.

[1] S. Perlmutter et al., Astroph. J. 517, (1999) 565; A. G. Riess et al. Astron. J. 116 (1998) 1009; A.G. Riess et al., Astroph. J. 560 (2001), 49; astro-ph/0207097; J.L. Tonry et al., Astroph. J. 594 (2003), 1; M. Tegmark et al., Phys. Rev. D 69 (2004), 103501.

[2] R.R. Caldwell, Phys. Lett. B 545, 23 (2002); S. Hannestad and E. Mörstell, Phys. Rev. D 66, 063508 (2002); M.P. Dạbrowski, T. Stachowiak and M. Szydłowski, Phys. Rev. D 68, 103519 (2003); P.H. Frampton, Phys. Lett. B 562 (2003), 139; S. Nojiri and D. Odintsov, Phys. Lett. B 562 (2003), 147; J.E. Lidsey, Phys. Rev. D 70 (2004), 041302.

[3] Hawking, S.W., Ellis, G.F.R., The Large-scale Structure of Space-time, Cambridge Univ. Press (1999) .

[4] J.M. Cline, S. Jeon, and G.D. Moore, hep-ph/0311312 I.Ya. Aref'eva, A.S. Koshelev, and S.Yu. Yernov, astro-ph/0507067.

[5] C. Csaki, N. Kaloper, and J. Terning, Ann. Phys. (N.Y.) 317 (2005), 410; astro-ph/0507148.

[6] H.K. Jassal, J.S. Bagla, T. Padmanabhan, Mon. Not. Roy. Astron. Soc. 356 (2005), L11; R.R. Caldwell and M. Doran, astro-ph/0501104

[7] V.K. Onemli, R.P. Woodard, Class. Quantum. Grav. 19 (2002), 4607; Phys. Rev. D70 (2004), 107301.

[8] E.R. Harrison, Nature 260 (1976), 591; P.T. Landsberg, Nature 263 (1976), 217; T. Chiba, Prog. Theor. Phys. 100 (1998), 1077; Yu. Shtanov and V. Sahni Class. Quantum Grav. 19 
(2002), L101; U. Alam, V. Sahni, T.D. Saini, and A.A. Starobinsky, Mon. Not. R. Astron. Soc. 344 (2003), 1057; V. Sahni, T.D. Saini, A.A. Starobinsky, and U. Alam JETP Lett. 77 (2003), 201; M. Visser, Class. Quantum Grav. 21 (2004), 2603.

[9] R.R. Caldwell and M. Kamionkowski, JCAP 0409 (2004), 009.

[10] M.P. Dąbrowski and T. Stachowiak, hep-th/0411199.

[11] J.D. Barrow, Class. Quantum Grav. 21, L79 (2004).

[12] J.D. Barrow, Class. Quantum Grav. 21, 5619 (2004).

[13] H. Štefančić, Phys. Lett. B586 (2004), 5; ibidem B595 (2004), 9; Phys. Rev. D71 (2005), 084024 .

[14] S. Nojiri and S.D. Odintsov, Phys. Lett. B595 (2004), 1; S. Nojiri, S.D. Odintsov and S. Tsujikawa, Phys. Rev. D 71 (2005), 063004.

[15] K. Lake, Class. Quantum Grav. 21 (2004), L129.

[16] J.D. Barrow and Ch. Tsagas, Class. Quantum Grav. 22, 1563 (2005).

[17] M.P. Dąbrowski, Phys. Rev. D71, 103505 (2005).

[18] L. Fernandez-Jambrina and R. Lazkoz, Phys. Rev. D70, 121503 (2004).

[19] A.G. Riess et al. Astrophys. J. 607 (2004), 665. 